\title{
Grupos domésticos socialmente vulnerables en el borde sur de la Zona Metropolitana de la Ciudad de México
}

\section{Socially vulnerable households in the southern edge of the Metropolitan Zone of Mexico City}

\author{
Perla Yannelli Fernández-Silva* \\ Blanca Rebeca Ramírez-VelázQuez**
}

\begin{abstract}
The latest studies about the relationship between city and countryside, regard the breakdown of the dichotomy among rural and urban. We present the results of a quantitative analysis in localities of the southern edge of the Metropolitan Zone of Mexico City. It identified social vulnerable households in transitional spaces between urban and rural, this vulnerability are constituted by socioterritorial characteristics which condition the forms of social and territorial reproduction of the groups in localities from Milpa Alta, Tlalpan and Tlábuac.
\end{abstract}

Keywords: territory, rural-urban relationship, social vulnerability, households.

\section{Resumen}

Los últimos estudios sobre la relación campo-ciudad señalan la ruptura de la dicotomía entre lo rural y lo urbano. En esta investigación se presentan los resultados de un estudio con metodología cuantitativa aplicado a localidades del borde sur de la Zona Metropolitana de la Ciudad de México, donde se identificó la existencia de algunos grupos domésticos en condiciones de vulnerabilidad social dentro de espacios de transición entre lo urbano y lo rural. Esta vulnerabilidad se constituye a partir de características socioterritoriales que, en conjunto, condicionan las formas de reproducción social y territorial de los grupos en localidades de Milpa Alta, Tlalpan y Tláhuac.

Palabras clave: territorio, relación campo-ciudad, vulnerabilidad social, grupos domésticos.

* Estancia posdoctoral de Conacyt en el Programa de Posgrado en Urbanismo de la UNAM México. Correo-e: perla.yannelli@gmail.com

** Universidad Autónoma de México, unidad Xochimilco. Correo-e: blare19@prodigy.net.mx 


\section{Introducción}

El crecimiento ampliado que ha tenido la Ciudad de México ${ }^{1}$ en las últimas décadas se caracteriza por una visión urbanocéntrica, en donde la expansión del tejido urbano se configura a partir de las zonas rurales que la circundan y conforman, lo que se considera en este trabajo, como los bordes de la Zona Metropolitana de la Ciudad de México. Esta expansión, caracterizada por algunos autores como dispersa (Pradilla-Cobos, 2011), se advierte como una transición en la que irremediablemente estas zonas se convertirán en urbanas.

Sin embargo, algunas consideraciones hacen suponer que existen otras formas de transitar de lo urbano a lo rural en las zonas metropolitanas, por ejemplo, la restricción normativa del uso de suelo de conservación, las características culturales asociadas con el hecho de ubicarse en zonas de pueblos tradicionalmente campesinos y con terrenos agrícolas de importancia en la zona.

En particular, en la porción sur del suelo de conservación de la Zona Metropolitana de la Ciudad de México se encontró que algunos grupos sociales, analizados en este trabajo, siguen reproduciendo su condición campesina, y otros, por el contrario, se insertan en mercados laborales netamente urbanos a pesar de vivir en condiciones eminentemente rurales. Algunos grupos domésticos no tienen condiciones para reproducirse social o territorialmente, lo que propicia que se encuentren en condiciones de vulnerabilidad social.

Los hallazgos antes mencionados constituyen aportes que permiten asumir que pueden cambiar las formas en las que se ha abordado la relación entre la ciudad y el campo. Sin duda este precedente ha generado que muchos autores consideren que existe una "nueva ruralidad" caracterizada por la necesidad de romper con la antigua forma en cómo se vinculaban lo rural y lo urbano.

Muchos autores desde diferentes posturas han tratado de resolver la dicotomía urbano-rural, desde los que tradicionalmente la han estudiado a partir de las diferencias demográficas, hasta los que analizan el diferencial entre las actividades económicas y los ingresos existentes entre ellas (De Grammont, 2005). También hay quienes tratan de ver las nuevas relaciones denominándolas "la rurbalidad" (Pacheco-Ladrón, 2002); otros asumen que en la actualidad se transita de una contradición a una complementariedad e hibridación entre las relaciones urbano-rurales (Méndez-Sastoque, 2005). Incluso están los que asumen que los cambios se deben al capita-

\footnotetext{
${ }^{1}$ Que corresponde con los límites político-administrativo del Distrito Federal (16 delegaciones), y no considera a los municipios conurbados del Estado de México, Hidalgo y Puebla de la Zona Metropolitana del Valle de México.
} 
lismo contemporáneo, y más que verlo como una novedad, es preciso estudiarlo como parte de un proceso en donde la historia tiene su cometido y la transición capitalista, ahora llamada por otros autores globalización o neoliberalismo, es necesario analizarse (Pradilla-Cobos, 2002).

Las visiones pueden ampliarse casi indefinidamente, y se encontrarán otras que enfatizan que las nuevas discusiones teóricas que hay sobre el espacio aportan evidencias importantes para considerar la eliminación de la dicotomía entre el campo y la ciudad (Barsky, 2013). Otra postura asume que se debería transitar a una visión en la que el análisis de las transformaciones urbanas y rurales se dan de manera conjunta, tanto en sus procesos de aceleración como en sus reticencias al cambio, que permite entenderlos tanto en la dimensión citadina como en la rural (RamírezVelázquez y Arias, 2002).

Cabe agregar, sin duda, algunas visiones teóricas que ven la relación desde la actuación del Estado en el espacio que definen como las periferias urbanas, en donde persisten y se encuentran pueblos que constituyen una forma específica de poblamiento y la planeación metropolitana los trata como urbanos, no obstante distan mucho de integrarse en una dimensión heterogénea que contribuya a su propia transición en relación con la ciudad (Cruz-Rodríguez, 2012).

En suma, si bien la "nueva ruralidad" favorece un panorama más amplio de la forma en cómo se analiza y percibe la tradicional relación campo-ciudad, es importante reconocer la necesidad de abrirse a estudios, que lejos de ser agrícolas o agrarios, incluyan dimensiones de análisis de las formas de reproducción económica y social que se manifiestan territorialmente en las zonas perimetrales de la ciudad, cuya complejidad rebaza las definiciones tradicionales de lo rural y lo urbano, al reconocer por un lado, las particularidades espaciales y, por el otro, la diversidad de agentes que se entrecruzan en las distintas actividades que ahí se desarrollan, sean éstas urbanas o rurales. Se produce con este reconocimiento, un espacio mucho más amplio y diverso del que tradicionalmente se había analizado en los procesos urbanos, con lo cual el espacio del borde se constituiría a partir de las formas simultáneas (Massey, 2005) de interacción diferencial que se generaran en el proceso de transformación de la zona metropolitana.

Así, sin ubicar este trabajo como parte constitutiva de la "nueva ruralidad", existe una motivación propia para analizar los procesos de transformación de las condiciones socioterritoriales de algunos grupos que habitan en el espacio rural o de conservación de la Ciudad de México, que coincide además, con los límites sureños de la Zona Metropolitana de la Ciudad de México (ZMCM). 
El objetivo de esta investigación es identificar las diferentes formas socioterritoriales que adoptan los grupos domésticos ubicados en las localidades del borde sur de la ZMCM en su organización y reproducción, y que en algunos casos, se hallan en una situación de vulnerabilidad social. Analizar la situación social de estos grupos y sus condiciones de vida es importante para que sean tomados en cuenta dentro de los procesos de gobernanza y de ordenamiento territorial que está llevando a cabo el gobierno citadino actual y las delegaciones en donde se realizó el estudio, así como para generar políticas de actuación sobre el suelo de conservación de la región.

Metodológicamente se analiza la relación del espacio rural con el tejido urbano en el borde sur de la ZMCM, tomando en cuenta los modos de interacción en las diferentes escalas socioespaciales que van desde la región metropolitana, hasta los espacios microsociales de los grupos domésticos ${ }^{2}$ a través de un estudio transversal cuantitativo, cuya recolección de datos se dividió en dos etapas.

La primera de ellas fue una revisión de información secundaria de los Censos de Población de 2000 y 2005 del Inegi, así como de imágenes satelitales. Para la selección de los sitios de estudio se consideraron variables como el tamaño de población, la actividad económica predominante, la localización geográfica, el año de formación de la localidad, el tipo de uso de suelo, entre otras. Los resultados arrojaron nueve localidades de tres delegaciones del borde sur de la ZMCM: San Francisco Tecoxpa, Zacaxontlicpa y Cazahuastitla, en Milpa Alta; San Miguel Topilejo, Parres y colonia La Venta, en Tlalpan; San Andrés Mixquic, colonia El Mirador y Zacatenco, en Tláhuac.

La segunda etapa consistió en la aplicación de instrumentos de investigación cuantitativa que permitieran ver a una escala menor los procesos microsociales de los grupos domésticos de las localidades seleccionadas, para ello se realizaron 270 encuestas amplias, determinadas por un muestreo estratificado proporcional ${ }^{3}$ que se desarrolló en 2007 y que consideraba multiples variables, por ejemplo, se investigó sobre las características generales y socioeconómicas del grupo doméstico, la práctica de actividades agropecuarias, las estrategias de organización interna y de apoyo entre los miembros, la ocupación principal, secundaria y eventual de cada uno de los integrantes, entre otras.

Los datos se procesaron estadísticamente para su interpretación y presentación en un trabajo de tesis doctoral que sirvió para una reflexión

\footnotetext{
${ }^{2}$ El grupo doméstico entendido como "el ámbito social en donde los sujetos organizan en armonía o en conflicto, diversas actividades necesarias para la reproducción de la vida inmediata, de manera diferencial entre el campo y la ciudad" (García et al., 1989: 168).

${ }^{3}$ Se consideró que el intervalo de confianza sería de $90 \%$ y un nivel de precisión de 5 por ciento.
} 
más específica de algunos aspectos identificados en esa investigación. Los resultados sugirieron desarrollar un análisis transdelegacional, por constituir un mejor nivel explicativo de la región, más que seguir la información disponible por las formas territoriales de carácter político-administrativo.

Metodológicamente se optó por el uso del concepto de borde, porque ofrece una alternativa libre de compromisos teóricos y permite la visibilidad de procesos que coexisten simultáneamente dentro de un mismo territorio. De igual manera, la inclusión de la vulnerabilidad social, como base para el análisis de las condiciones socioeconómicas y culturales del tipo de grupo doméstico que aquí se presenta, permite centrarse en la inseguridad e indefensión de los grupos ante condiciones de desventaja, ocasionado por el modelo de desarrollo metropolitano del capitalismo neoliberal. Pero también permite vislumbrar estas zonas en su dimensión multidimensional al conjuntar los procesos diversos que ahí se desarrollan (Massey, 2005), sin que irremediablemente estén supeditados a las condiciones urbanas o al centro histórico, como lo hacen otras posturas (Ramírez-Velázquez, 2007) que se analizarán más adelante.

Durante el proceso de investigación se eligió el uso de la categoría de grupo doméstico como objeto de estudio, porque representa la unidad social mínima en donde se lleva a cabo cotidianamente la continuación del sistema social por parte de cada uno de los agentes sociales.

A diferencia de los conceptos metodológicos de familia y hogar, el grupo doméstico incorpora al análisis los temas de producción y reproducción social (Chayanov citado en De Oliveira et al., 1989), y advierte sobre la necesidad de vincular las actividades de producción y consumo en la investigación, así como analizar las interrelaciones entre los miembros del grupo con la unidad productiva. Estos grupos habitan en localidades que el Censo (Inegi, 2005) caracteriza como rurales y dentro del territorio definido como borde sur de la ZMCM. Si bien algunos no están directamente insertos en la dinámica de reproducción rural, su localización de borde en relación con la ciudad y su condición de colonias o de asentamientos cercanos a las localidades, permiten evidenciar su participación en la transición rural-urbana de estas zonas, o bien, de la simultaneidad de procesos que ahí se presentan y que complejizan su caracterización y su condición dentro de la ciudad.

La vulnerabilidad social de los grupos domésticos del borde sur de la ZMCM se manifiesta especialmente en localidades dentro de territorios históricamente agrícolas -con características culturales y tradiciones que datan de la época prehispánica- que se han ido incorporando a la nueva dinámica urbana paulatinamente y de forma muy diversa y específica, dependiendo de las condiciones en las que se encuentren en cada zona. 
Los hallazgos de esta investigación que definen a algunos grupos como socialmente vulnerables se argumentan a partir de cinco ejes de análisis: el tipo de estructura del grupo doméstico, el origen de los jefes de familia, el tipo de propiedad del suelo en el que habitan, el vínculo con suelo agropecuario y el tipo de ocupación o empleo. Todos estos puntos permiten hacer visible a un segmento de la población cuyas características socioeconómicas evidencian la diferenciación socioespacial al interior de las localidades del borde sur de la ZMCM.

\section{El borde sur de la ZMCM}

El concepto de borde constituye metodológicamente la franja variable que circunda a la mancha urbana consolidada, y que es parte medular de su transición, crecimiento y evolución (Ramírez-Velázquez, 2007). Se refiere al límite entre lo urbano y lo rural, pero no como línea divisoria y terminal sino como el resultado del análisis de los procesos que ahí se desarrollan y que pueden ser caracterizados de una u otra manera; se visualiza como una zona de transición, de fronteras o de nuevos procesos, con la intensión de no invisibilizarlos o subordinarlos.

El borde trasciende el debate de los acotamientos, es decir, se va directamente a analizar los procesos que suceden en los márgenes de las ciudades sin tener que hacer referencia obligada a un centro o una centralidad específica, la cual se da por entendido que existe, ya que es parte de su esencia pero no la parte fundamental de su definición o delimitación (Ramírez-Velázquez, 2007). Pero también va más alla de los límites entre lo urbano y lo rural, ya que en el borde sur se traslapan procesos que pueden ser urbanos y rurales a la vez en un mismo territorio. El borde permite concebir un espacio abierto y permeable, para reconocer que existen relaciones externas a él que lo configuran y lo moldean desde diferentes escalas y posicionamientos, por lo que se encuentra en una continua y constante transición o cambio, en la medida en que se relaciona con la ciudad.

El reconocimiento de la heterogeneidad es intrínseca en su definición, ya que los bordes se generan por las prácticas de construcción de la ciudad y del campo y se presentan como zonas de interacción de varias actividades, por lo tanto, la diversidad y la complejidad son sus características fundamentales (Ramírez-Velázquez, 2007) que se transforman cotidianamente, ya que el cambio y el movimiento son inherentes al territorio.

Geográficamente, el borde sur se conforma por el suelo no urbano de las delegaciones Tlalpan, Milpa Alta y Tláhuac, y corresponde con 31\% de la superficie total de la ZMCM. Del total de la superficie de cada 


\section{Figura 1}

\section{El borde sur de la ZMCM}

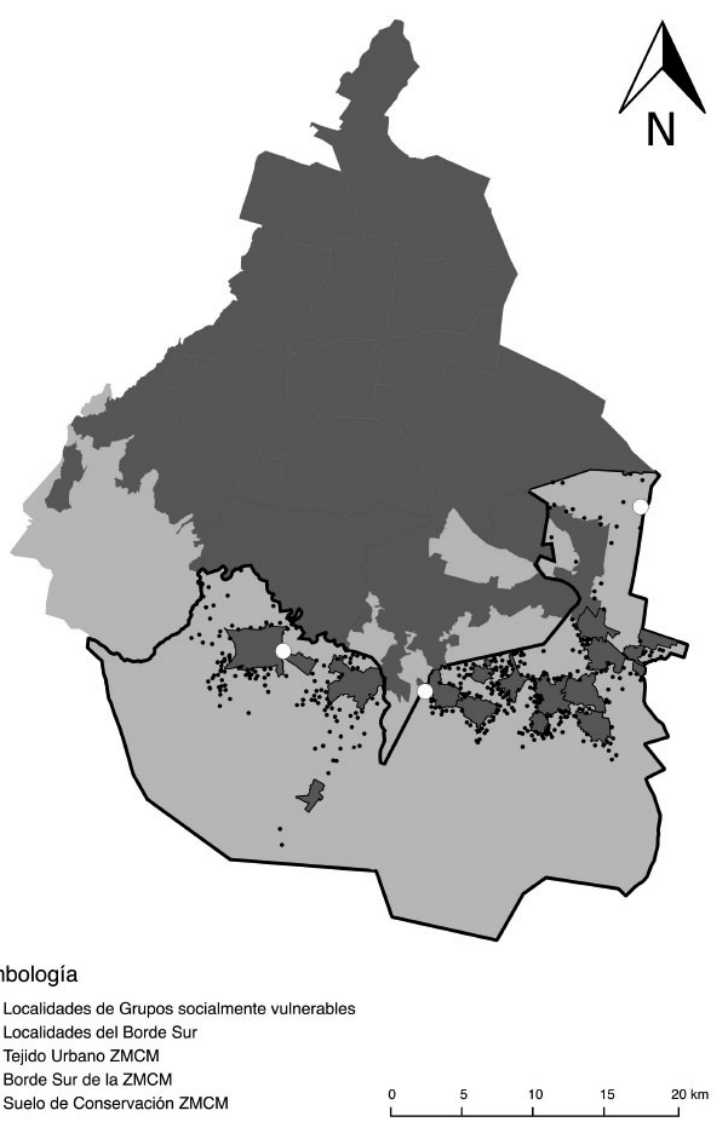

Fuente: elaboración propia con datos del Inegi 2010.

delegación $72 \%$, 80\% y 93\% son de uso no urbano ${ }^{4}$ para Tláhuac, Tlalpan y Milpa Alta, respectivamente. El borde sur del Distrito Federal, que es a su vez el sur de la Zona Metropolitana de la Ciudad de México, está formado por una gran zona decretada como de conservación y preservación ecológica que mantiene características rurales variadas, no sólo por su conformación física territorial, sino en gran parte por sus formas socioculturales, debido a su carácter histórico que se mantiene vigente a pesar de la gran influencia cultural de la urbe.

${ }^{4}$ En el interior de estas unidades administrativas, la superficie no urbanizada está destinada al uso de suelo de preservación y rescate ecológico, producción rural agroindustrial, zona agropecuaria y áreas de preservación. 
Más que referirse a un espacio específico con condiciones ambientales particulares dignas de conservar y dirigir su desarrollo, hace referencia, además, a la multiplicidad de procesos que coexisten en espacios sociales bajo condiciones, históricas y productivas muy específicas y que se mantienen en constante interacción con procesos externos a él, que lo reconstruyen y transforman continuamente, por lo tanto se reconocen los cambios, porque le proporciona elementos dinámicos de análisis, como la historia y la multiplicidad productiva del espacio social.

Territorialmente, el borde sur está constituido por pequeños asentamientos dispersos que dan una apariencia fragmentada a la mancha urbana, un tejido que se diluye y se entremezcla con terrenos baldíos, milpas y campos. Estos núcleos o asentamientos presentan particularidades socioterritoriales caracterizadas por el tamaño de su población, extensión y conformación territorial, actividades productivas y características históricas y culturales. Si bien existen múltiples asentamientos (figura 1) cuyo origen y configuración territorial es diversa, un aspecto en común es que la mayoría de ellos tienen menos de 2500 habitantes, con una morfología poco compacta.

Desde un enfoque ${ }^{5}$ de proximidad y distancia respecto a los núcleos urbanos, las localidades del borde pueden caracterizarse bajo las siguientes dos categorías: localidades alejadas de la mancha urbana y las cercanas. El criterio para estar dentro de una u otra, principalmente, es la distancia respecto al tejido urbano en un rango de cinco kilómetros (FernándezSilva, 2011), lo que marca y define las formas de relación que se establecerán entre las pequeñas localidades y los núcleos urbanos más consolidados y que se desarrollan más adelante en este trabajo. Los grupos domésticos que fueron analizados corresponden con el tipo de localidad cercanas al tejido urbano.

Si bien cada localidad presenta particularidades en sus procesos internos, tienen en común elementos como la conservación de tradiciones y la cosmovisión propia de estos sitios que están relacionadas con la cotidianidad de los pobladores, es decir, con el día a día de las actividades que se realizan; por lo que no es difícil ver que parte de los grupos domésticos se dedican a actividades agropecuarias y han encontrado la forma de insertarse en una dinámica compleja que se presenta no sólo en la escala regional metropolitana, sino en el nivel nacional y global.

En el borde sur se identifica la readecuación socioeconómica de muchas de las localidades rurales del sur de la ZMCM al entretejerse, readecuarse y yuxtaponerse con dinámicas socioespaciales aceleradas propias y carac-

\footnotetext{
${ }^{5}$ Se reconoce que existen múltiples criterios para caracterizar a las localidades del borde sur de la Ciudad de México, sin embargo, el criterio que guió este trabajo de investigación se inscribe bajo un enfoque teórico que aborda la relación campo-ciudad desde una visión de bidireccionalidad.
} 
terísticas de la ciudad, que es necesario reconocer y analizar. Por lo tanto, es importante preguntarse ¡cómo se insertan socioeconómicamente los grupos domésticos del borde sur en territorio particularmente agropecuario? y ¿qué sucede con aquellos grupos que no se integran a esta dinámica agrícola? La respuesta a estas interrogantes es parte del análisis que se desarrolla en la tercera parte de este trabajo.

\section{La vulnerabilidad social}

El concepto de vulnerabilidad por lo general se asocia con una idea de riesgo para la población ante desastres naturales, sobre todo, cuando la afectación más significativa es para las poblaciones más pobres, aquellas cuyos soportes materiales como la vivienda y la ocupación irregular del suelo, no apto para uso habitacional, ponen de manifiesto su fragilidad e incapacidad para enfrentar los impactos urbanos o ambientales generados por eventos naturales como lluvias, inundaciones, temblores, ciclones, entre otros (Ramírez-Velázquez, 2012).

Bajo los fundamentos de inseguridad, debilidad y desventaja frente al peligro en interacción con el entorno, la vulnerabilidad se convierte en una "condición dinámica" (Macías citado en Ramírez-Velázquez, 2012). Dos décadas más tarde al concepto de vulnerabilidad se le añadió el adjetivo "social" para ampliar la visión y permitir describir las condiciones de indefención, fragilidad e incapacidad de los territorios, las regiones y de los grupos sociales ante el desarrollo neoliberal, cuyas repercusiones en las formas de producción y soportes materiales ha generado altos índices de desigualdad y exclusión social en América Latina. Por ejemplo, en México el abandono del desarrollo del campo, la contaminación y extracción de recursos naturales, el proceso de desindustrialización, la precariedad del empleo del sector terciario (Ramírez-Velázquez, 2012) y la dependencia del crecimiento económico con los mercados globales han contribuido con la creciente vulnerabiliad de los territorios y regiones. Por lo tanto, la vulnerabilidad social se convierte en:

... un rasgo específico de la forma que ha adoptado el capitalismo en los últimos años: economía de libre mercado, abierta al mundo y con Estado mínimo. Así como en el pasado la denominada industrialización por sustitución de importaciones tuvo en la marginalidad su fenómeno social más distintivo, en el actual período histórico la vulnerabilidad social aparece como el rasgo dominante del patrón de desarrollo vigente (Pizarro-Hofer, 2001: 7).

Frecuentemente se identifica la condición de pobreza con vulnerabilidad. Sin embargo, la inseguridad e indefensión que caracterizan a esta última no son necesariamente atribuibles a la insuficiencia de ingresos, 
propia a la depauperación de la primera (Pizarro-Hofer, 2001). En contraposición, en un intento por comprender las condiciones sociales que se viven en América Latina, la Cepal (1998), con base en la experiencia europea, utilizó el enfoque de exclusión para denominar a la población cuyas condiciones sociales no se integraban al sistema económico; sin embargo, este concepto no es transferible a una realidad como la latinoamericana que tiene una posición diferente en la dinámica económica global. Por el contario, las nuevas políticas y modelos de desarrollo incluyente han tratado de integrar, vía el desarrollo de las comunicaciones y mercados locales, a los países latinoamericanos al modelo económico neoliberal, cuyos resultados sólo han dejado ver de manera más clara la desigualdad entre las regiones (Pizarro-Hofer, 2001) y la vulnerabilidad social en la que se encuentran los sectores insertos a dicho modelo.

La vulnerabilidad social en el territorio aplica tanto para espacios urbanos como rurales, "si se comparan las condiciones de vida de los trabajadores urbanos con la de los campesinos de áreas remotas es probable que éstos, al basar su vida en la agricultura de subsistencia, se hayan visto menos afectados frente a los programas de ajuste estructural y macroeconómicos" (Pizarro-Hofer, 2001: 8). En cambio, los trabajadores urbanos, al depender predominantemente de los ingresos provenientes del empleo y enfrentarse a los desafíos de las nuevas instituciones y reglas del juego del patrón de desarrollo vigente, se encuentran más expuestos a la vulnerabilidad social aún cuando no siempre estén bajo la línea de pobreza (Pizarro-Hofer, 2001).

Para el borde sur de la ZMCM, la reproducción de los grupos domésticos, a través de la actividad agropecuaria, está mediada por la interacción de un conjunto de variables como el tipo de localidad, tipo de tenencia de la tierra, la estructura del grupo doméstico y el lugar de origen del jefe de familia, principalmente. En contraposición, se encuentran aquellos núcleos familiares, que a pesar de estar en espacios rurales en condiciones altamente propicias para reproducirse como campesinos, y en convivencia con otros grupos vecinos dedicados a la producción agrícola, se insertan en una dinámica laboral metropolitana en condiciones precarias, complejas e inseguras.

Los grupos domésticos socialmente vulnerables se definen como el conjunto de individuos vinculados o no por lazos de consanguinidad y de convivencia dentro de una misma vivienda, cuyas formas de trabajo los colocan en una posición de riesgo ante una dinámica socioeconómica metropolitana desventajosa y desigual. Las formas de interacción socioterritoriales de estos grupos no les aportan seguridad o solidez para resistir situaciones socioeconómicas críticas. 
Por otro lado, las características socioespaciales que los determinan no les dan las posibilidades, ni a corto ni a largo plazo, de implementar estrategias alternativas que les permitan transformar las condiciones de supervivencia. Por lo tanto, si los grupos están en una condición de "vulnerabilidad social", significa que no son lo suficientemente capaces de crear las condiciones materiales que les permita transformar sus condiciones de vida actuales a otras mejores, dentro de espacios rurales en donde la actividad agropecuaria puede ser una opción de forma productiva que les dé mayor seguridad e independencia, incluso a pesar de su intención de no reproducir su modo de vida rural.

Las características de los grupos socialmente vulnerables del borde sur de la ZMCM (Fernández-Silva, 2011) se encuentran definidos bajo los siguientes ejes de análisis socioterritorial:

a) Son grupos domésticos con estructura monoparental con jefatura femenina; ahí se alojan madres jefas de familia, quienes son las directamente responsables de sobrellevar la carga del mantenimiento de la casa, la familia y del trabajo doméstico.

b) Una mayor proporción de jefas de familia originarias de provincia. En ese sentido representan una parte de la migración del campo a la ciudad.

c) Se encuentran con inseguridad legal de la propiedad de sus viviendas. No tienen escrituras y ocupan espacios con uso de suelo no urbano. En general, son asentamientos irregulares al ocupar zonas decretadas de conservación, o bien, localidades que se forman por invasiones o por otras condiciones ilegales de tenencia de la tierra.

d) No poseen terrenos de cultivo, ni tienen la posibilidad de acceder a ellos.

e) Su estructura ocupacional es precaria, con un alto desgaste físico y mal retribuida.

A partir de este estudio se encontró que existen grupos domésticos que están altamente integrados a la dinámica agropecuaria del borde sur de la ZMCM. En el cuadro 1, puede observarse que 25,41\% de los jefes de familia se vinculan a través de la activiad agrícola con el territorio por la posesión de un terreno de cultivo, independientemente de que esté en uso o no. De la población encuestada, $15,41 \%$ se dedica a trabajar en el sector primario de la economía. 


\section{Cuadro 1 \\ Porcentaje de jefes de grupos domésticos de localidades del borde sur de la ZMCM vinculados con el sector agropecuario}

\begin{tabular}{lccccc}
\hline \multicolumn{5}{c}{ Ocupación principal por sector } \\
\hline & Primario & Secundario & Terciario & Total uso \\
\hline $\begin{array}{l}\text { Tienen terreno de } \\
\text { cultivo }\end{array}$ & Uso actual & 8,75 & 2,50 & 9,58 & 20,83 \\
& No uso & 0,00 & 0,41 & 4,16 & 4,58 \\
$\begin{array}{l}\text { No tienen terreno } \\
\text { de cultivo }\end{array}$ & 6,66 & $\mathbf{1 8 , 3 3}$ & $\mathbf{4 9 , 1 6}$ & $\mathbf{7 4 , 1 6}$ \\
\begin{tabular}{l} 
Total por sector \\
\hline
\end{tabular} & 15,41 & 21,25 & 62,62 & 100,00 \\
\hline
\end{tabular}

Fuente: Fernández-Silva, 2011.

Cabe mencionar que $67,49 \%$ del total de los jefes de los grupos domésticos del borde sur de la ZMCM no se insertan en una dinámica propiamente agrícola o ganadera porque no tienen un terreno para cultivo y trabajan en los sectores industrial o de comercio y servicios. De ese porcentaje, sólo $13,33 \%$ presentaron características que los tipifica como grupos socialmente vulnerables.

Estos grupos se localizan específicamente en las localidades de El Mirador en Tláhuac, colonia La Venta en Tlalpan y Cazahuastitla en Milpa Alta. Son pequeñas "colonias" contiguas a localidades urbanas más grandes y consolidadas que cuentan con todos los servicios e infraestructura como centros de salud, tianguis o mercados, además de accesos rápidos a vías principales de transporte. Los grupos que aquí describimos hacen uso del equipamiento de las localidades urbanas vecinas; sin embargo, oficialmente no pertenecen a ellas.

Estas localidades se formaron en la década de 1990, por ello nueve de cada diez familias tienen menos de veinticinco ańos viviendo en estos asentamientos. La forma en la que adquirieron sus terrenos fue a través de la compra ilegal de lotes a un particular ya que estos predios no se pueden ni comprar ni vender porque el Gobierno del Distrito Federal, a través de la Secretaría de Ordenamiento Territorial, decretó ${ }^{6}$ a la localidad en el 2000 como parte del Suelo de Conservación. La misma ilegalidad ocasionó que la adquisición de predios fuera a precios muy bajos.

\footnotetext{
${ }^{6}$ El Programa General de Ordenamiento Ecológico del Distrito Federal (PGOEDF). Se aprobó el 28 de abril del 2000. Sin embargo, varios años atrás ya se estaban realizando las gestiones (Gobierno del Distrito Federal, Secretaría del Medio Ambiente y Comisión de Recursos Naturales, 2000).
} 
A continuación se analizan las cinco características socioterritoriales que algunos de los grupos domésticos ${ }^{7}$ de las localidades del borde sur poseen y que los coloca en una posición de vulnerabilidad social en comparación con otros tipos de grupos domésticos de la región.

\section{Los grupos domésticos socialmente vulnerables}

\subsection{Estructura endeble del grupo doméstico}

Los grupos domésticos organizan en armonía o en conflicto diversas actividades necesarias para la reproducción de la vida inmediata, en función del tipo de arreglo y negociación interna entre sus integrantes, esto con base en el número de miembros, el tipo de relación consanguínea que existe y las relaciones de poder que se ejercen entre los agentes que lo conforman (Chayanov citado en De Oliveira et al., 1989).

En este trabajo se observó que el tipo de grupo doméstico más común (65\%) en las localidades del borde sur, es el nuclear biparental (estructura familiar de padre, madre e hijos), que permite la reproducción de los grupos a través de la repartición más o menos equitativa de las tareas y actividades cotidianas dentro de los ámbitos del hogar y del trabajo. Sin embargo, los grupos domésticos socialmente vulnerables son del tipo nuclear monoparental con jefatura femenina, quienes han encontrado condiciones propicias para su reproducción dentro de estos espacios.

La evidencia mostró, además, que los factores asociados al tipo de localidad en el que habitan les permite a estas jefas de familia acceder de forma ilegal a la compra o renta de un lote; entre éstos: los bajos costos del suelo, la poca presión social en contra de las mujeres cabezas de familia, el rápido acceso a los servicios urbanos y centros de trabajo, entre otras, son algunas de las principales características que hacen a estos asentamientos, lugares propicios para que los grupos nucleares con jefatura femenina elijan establecerse en ellos.

En lo que respecta al trabajo que realizan, que se explicará más adelante, la mitad de las mujeres que encabezan un hogar trabajan como empleadas domésticas, mientras que en tres de cada diez casos son obreras; es decir, la probabilidad de que las mujeres jefas de familia accedan a un trabajo bien remunerado de alta calificación es muy poca, esto se debe a que no cuentan con una educación escolarizada o preparación profesional: por ejemplo, 30\% de ellas estudió únicamente la primaria, $50 \%$ algún año de secundaria, el resto, sólo $20 \%$, alguna carrera técnica.

7 Los resultados de grupos vulnerables que se presentan a continuación corresponden a 32 grupos domésticos analizados para las localidades de El Mirador, en Tláhuac; colonia La Venta, en Tlalpan, y Cazahuastitla, en Milpa Alta, de un total de 270 grupos domésticos encuestados. 
Sin embargo, llama la atención el hecho de que localidades más grandes y pobladas del borde sur de la ZMCM no sean espacios elegibles para el asentamiento de los grupos vulnerables. Se considera que, la cohesión social y el fuerte conservadurismo de las tradiciones culturales de los pueblos, aunado a las pocas posibilidades de acceder y conservar un terreno, son factores determinantes para que las jefas de familia busquen las localidades del borde sur cercanas a poblaciones urbanas consolidadas para establecer su residencia.

Se caracterizan por ser estructuras familiares que amparan a un mayor número de niños y adolescentes que, por su edad, no aportan recursos al gasto de los grupos, pero que son consumidores importantes por estar en edad escolar. Lo anterior podría explicarse por la falta de capital de inversión, poca capacitación y organización interna de los grupos, todo ello, debido a sus antecedentes como inmigrantes, su escaso patrimonio, y el requerimiento inmediato de satisfacción de necesidades básicas.

\subsection{Jefas de familia originarias de provincia}

La mayoría de los grupos domésticos socialmente vulnerables se caracterizan porque su jefa de familia proviene de otros estados de la república. Para Milpa Alta y Tláhuac, alrededor de 65\% son foráneas, mientras que para Tlalpan es casi $40 \%$, lo que expresa un movimiento de migración originada por la falta de opciones de vida en alguna otra parte del país (cuadro 2).

\section{Cuadro 2}

\section{Porcentaje de lugar de nacimiento de las jefas de familia de grupos vulnerables de las localidades del borde sur}

\begin{tabular}{lccc}
\hline \multicolumn{1}{c}{ Lugar de nacimiento } & Milpa Alta & Tláhuac & Tlalpan \\
\hline Fuera del Distrito Federal & $63,33 \%$ & $66,66 \%$ & $36,67 \%$ \\
Distrito Federal & $36,66 \%$ & $33,33 \%$ & $63,33 \%$ \\
Total & $100,0 \%$ & $100,0 \%$ & $100,0 \%$ \\
\hline
\end{tabular}

Fuente: elaboración propia con datos de Encuesta "Grupos domésticos en el borde sur de la ZMCM".

Las jefas de familia que nacieron en provincia, en seis de cada diez casos, trabajaron en actividades vinculadas con la agricultura hace más de veinte años; es decir, su origen fue campesino y ante la escasez de opciones, optaron por migrar a la Ciudad de México en búsqueda de mejores oportunidades de trabajo. Cuando llegaron a la urbe, sus condiciones de vida eran complejas y precarias ya que en más de $80 \%$ de los casos no 
contaban con ningún familiar o conocido como apoyo, no tenían una vivienda asegurada ni la posibilidad de adquirir un terreno de cultivo para continuar desarrollando la actividad agropecuaria.

La Ciudad de México constituyó un territorio idealizado por las jefas de familia de los grupos domésticos vulnerables, bajo el supuesto de la búsqueda de una inserción laboral no agrícola como la mejor opción ocupacional y residencial, y la más atractiva oportunidad para elevar sus niveles de vida (Negrete, 2000; Skewes, 2014). Así, estas jefas de familia salieron de sus lugares de origen hace veinte o veinticinco años, dejando atrás muchas de sus estructuras sociales que les proporcionaban arraigo y cohesión social.

A pesar de lo anterior, se percibió que estos grupos ya están en una etapa de estabilización (Adler de Lomnitz, 1997), es decir, ya ocurrió un proceso de reestructuración social que les ha proporcionado una nueva visión de la realidad urbana. Sin embargo, esta adaptación al entorno implica el rompimiento con muchas estructuras campesinas, la modificación o adaptación de prácticas cotidianas al nuevo espacio de residencia y el apoyo o vinculación con formas específicas de la dinámica social de la urbe que les permite ahora reproducirse como grupo.

Al llegar a la ciudad las opciones laborales en las que pudieron insertarse dependían de su escolaridad, capacitación previa y habilidades personales. Debido a su origen campesino, los tipos de empleo a los que se incorporaron fueron los de baja calificación y depreciada remuneración, ya que no eligieron trabajar en actividades agrícolas en los bordes de la ciudad. Con el tiempo, estas jefas de familia llegaron a formar grupos que demandaban condiciones de vida distintas a las que hasta ese momento se habían proporcionado. Todo esto apunta a que el análisis de la estructura del grupo doméstico es fundamental para comprender las formas de organización internas y su vinculación con el territorio.

\subsection{Inseguridad con la propiedad de la tierra}

La condición de vulnerabilidad social considera también los derechos que cada ciudadano tiene para contender con su transformación y desarrollo integral como ser humano. Para el caso de las mujeres de grupos sociales con menor poder social, también se considera la posilibidad del acceso a la propiedad, al mercado laboral, a los ingresos y recursos que condicionan su derecho a la ciudad (Czytajlo citado en Ramírez-Velázquez, 2012). Bajo esta premisa, los grupos domésticos socialmente vulnerables del borde sur de la ZMCM no cuentan con un documento legal que ampare la propiedad del terreno en el que viven, ya que sólo cuentan con un recibo de compra-venta entre particulares. Lo anterior se debe a la incom- 
patibilidad con el uso del suelo oficial de reserva ecológica que se generó a partir de la venta ilegal de los antiguos propietarios al ver la pérdida de sus tierras agrícolas ante el inminente cambio de uso del suelo.

La solución al problema no parece cercana ni fácil ya que varias autoridades están involucradas. Por ejemplo, la Dirección General de la Comisión de Recursos Naturales (Corena) acordó desde el 2000 en algunas localidades (Milpa Alta) la incorporación al programa de crecimiento cero, en el cual los pobladores se comprometían a no incrementar la superficie construida y a desempeñar actividades agrícolas en los solares de sus terrenos. Otro caso, ha sido el reconocimiento de las Delegaciones administrativas (Tlalpan y Tláhuac) de estos asentamientos con el único compromiso de proporcionar agua potable gratuita y limitada a cada una de las viviendas de estas localidades.

Por lo pronto no se vislumbra, al menos en el corto plazo, una solución ya que el reconocimiento e inclusión de estas localidades al llamado "casco urbano" por parte de las Delegaciones administrativas pone en riesgo el crecimiento e invasión de estas zonas ecológicas. Por lo anterior, se considera que el Gobierno del Distrito Federal priorizará la conservación de los ecosistemas de la ciudad a costa de preservar la falta de servicios. Esta situación de anomalía legal conlleva a que las localidades del borde sur no cuenten con la dotación de servicios públicos básicos, por ejemplo, las calles no están pavimentadas, no hay drenaje, y sólo en la localidad de El Mirador Tláhuac, las viviendas cuentan con agua entubada en su interior, el suministro de energía eléctrica es inestable ya que la mayoría de las familias están conectadas de manera irregular, de tal suerte que las condiciones de vida de la población de estas localidades es similar al modo de vida del campo, con la diferencia de su "rápido" acceso y disposición de servicios en los pueblos cercanos a los que se encuentran.

Las viviendas, por lo general, ocupan sólo una porción del terreno en el que habitan, ya que en la mayoría de los casos se trata de una sola casa por terreno. En el resto del espacio, puede observarse material de construcción regado o en espera de ser colocado, algunos pedazos de concreto que evitan el contacto directo con la tierra y que señalan los accesos a las casas, botes grandes de plástico o metálicos que llenan con la pipa les surte de agua una vez a la quincena. En tres de cada diez casos, tienen techos de lámina y, al menos, entre una y tres tienen piso de tierra. Siete de cada diez viviendas son de un sólo nivel y en general son de tipo progresivo (Villarreal-González y Castańeda, 1986), entendida como el tipo de vivienda que se construye lentamente, durante lapsos largos, por lo que las familias viven en construcciones en obra negra y poco a poco, conforme a como sus recursos se lo permiten, van completando los procesos constructivos. 
Una de cada diez casas son un cuarto redondo, y entre $73 \%$ y $76 \%$ tienen un sólo cuarto para dormir. Como se puede advertir, las condiciones de vida material de estas localidades son precarias, y los colocan en una situación social vulnerable; sin embargo, ¿por qué este tipo de grupos domésticos habitan estos espacios?, ¿qué características tienen estos terrenos para que sean accesibles para ellos?, ¿por qué dentro de todos los espacios de la ZMCM estas localidades periféricas están disponibles?

El principal factor es el costo del suelo, la ilegalidad de la propiedad asociada a la escasez de servicios públicos abarata el precio de los lotes. Por ejemplo, en Milpa Alta, en 2006 un terreno valía 370 pesos el metro cuadrado si se considera que, en promedio, el precio de un terreno sin servicios con un uso de suelo urbano era de mil pesos (Fernández-Silva, 2011) para ese mismo año y esa misma zona, la condición de ilegalidad abarata alrededor de $63 \%$ el costo del terreno, lo que posibilita que una familia con esfuerzo pueda comprar un lote dentro de estas localidades con las características anteriormente descritas. Cabe señalar que en estas localidades el impuesto sobre el uso de la tierra o Predial no existe, lo que abarata además el costo de vida en estos espacios, a partir de la evasión o excención de impuestos.

Finalmente, a pesar de ocupar un terreno irregular, incierto, y de carácter provisional, los grupos domésticos de estas localidades han construido y levantado sus casas con el mayor empeño que les ha sido posible y han luchado por gestionar el cambio de uso del suelo. Con el transcurrir del tiempo los pobladores se han arraigado a sus colonias y las mejoras ya son tangibles según sus propios habitantes. A pesar de lo anterior, existe un grupo de pobladores de las localidades urbanas contiguas que luchan en contra de todas estas mejoras ya que se sienten amenazados ante la posibilidad de invasión de sus terrenos de cultivo próximos a estas localidades.

\subsection{Dificultad para acceder al uso de tierras de cultivo}

Si estos grupos domésticos vulnerables no tienen certeza por la propiedad de sus terrenos habitacionales, ya que con grandes esfuerzos cubrieron su costo, más complejo sería para ellos adquirir un espacio de tierra para desarrollar actividades agropecuarias que pudieran complementar sus bajos salarios. La disponibilidad de tierras de cultivo en estas zonas es escasa puesto que los agricultores tradicionales tienen bien controlado el mercado de tierras y, en algunos casos, (10\%) tienen un carácter de tipo ejidal o comunal. Al parecer no existe una demanda representativa de tierras por parte de estos grupos, es decir, no hay un interés tangible por 
adquirir espacios para la agricultura. Por lo tanto, se puede decir que no tienen la intención de reproducirse como campesinos.

A pesar de que el campo y la actividad agrícola circunda a las localidades del borde sur que se analizaron, no integran a los miembros de los grupos domésticos socialmente vulnerables, ellos conviven y se desplazan en ambientes de carácter campesino sin involucrarse directamente en esta actividad. Por ello, las proporciones de Población Económicamente Activa en el sector primario para estas localidades es de apenas uno por ciento.

El hecho de que los grupos vulnerables de dichas localidades no cuenten con un terreno de cultivo, sólo es un factor, entre otros, que impide que se reproduzcan como grupos campesinos, pues se considera que deben darse ciertas condiciones para que la actividad agropecuaria se realice, como un conjunto de personas ya sean familiares o empleados. Por lo tanto, la agricultura o ganadería no es una opción de vida para estos grupos vulnerables en la cercanía de la ciudad, en cambio, sus opciones de trabajo las encuentran en sectores que requieren baja capacitación y por lo tanto son poco remunerados.

\subsection{Estructura ocupacional precaria}

A pesar de que el modelo económico neoliberal ha permitido la apertura de los mercados al mundo. El repliegue del estado de las funciones propiciaron nuevas y mayores oportunidades profesionales y de negocios a varios sectores de la población mundial. Pero, por otra parte, también provocaron un cambio tan rápido en la economía y en las instituciones que acentuaron las condiciones de vulnerabilidad de las personas y familias de ingresos medios y bajos en las áreas urbanas, quienes se encuentran hoy expuestas a mayores riesgos de los que existían bajo el patrón de desarrollo de la sustitución de importaciones (Pizarro-Hofer, 2001).

En el caso de las jefas de familia de los grupos domésticos vulnerables del borde sur de la ZMCM los bajos niveles de escolaridad ( $2^{\circ}$ año de secundaria, en promedio) aunado a la falta de opciones y posibilidades de incrementear su capacitación y entrenamiento les impide incorporarse a sectores productivos mejor remunerados. Si se considera que las instituciones y empresas solicitan trabajadores para los puestos más básicos a personas con nivel escolar medio superior, estas jefas de familia no pueden acceder a ellos. Por lo tanto, se emplean en trabajos que implican un mayor trabajo físico que mental, con las repercusiones en la salud asociados a este tipo de empleos.

De acuerdo con lo anterior, los trabajos más frecuentemente desempeñados son como obreras (13\%) o trabajadoras domésticas (9\%); en ambos casos la capacitación es mínima o nula. Es importante señalar que 
las localidades con mayor proporción de jefas de hogar obreras viven en las localidades analizadas de Tláhuac y Tlalpan, mientras que las trabajadoras domésticas residen en los espacios del borde sur de Tláhuac y Milpa Alta, principalmente. Otros empleos en los que se ocupan las jefas de familia de los grupos domésticos socialmente vulnerables también son de baja remuneración e inseguridad laboral como el comercio informal, ya que son trabajos que dependen de la temporalidad. Por ello, el ingreso superior que reciben en un día compensa aquellos en los que no encuentran trabajo o no venden sus productos.

Como parte de las hipótesis iniciales del estudio, se esperaba que un gran porcentaje de jefas del grupo doméstico buscaran otras fuentes de recursos, es decir, trabajos complementarios que les ayudaran a obtener un mejor ingreso. Las evidencias empíricas demostraron lo contrario, y se explica a partir del analisis que el tiempo destinado a su principal ocupación de ocho horas en promedio, aunado a los tiempos de transporte y las actividades de reproducción o de atención al hogar, ocupan el total del tiempo de las jefas de familia sin considerar el desgaste físico asociado.

La importancia de la estructura del grupo doméstico resalta la posibilidad o no de hacerse de otro ingreso que complemente al de la jefa de familia. Por ejemplo, sólo en uno de cada diez de los grupos vulnerables, algún hijo trabaja. Por ello, los grupos domésticos nucleares monoparentales se consideran los grupos más vulnerables ya que sólo la jefa de familia estaría en posibilidades de asegurar el ingreso del grupo, además de desempeñar las actividades cotidianas de reproducción del hogar.

Contar con un trabajo de tipo asalariado implica la seguridad diaria de recibir un ingreso por una jornada, bajo una relación laboral patrónempleado. Por lo tanto, estos altos porcentajes de jefas de grupo doméstico vulnerables que trabajan en este tipo de empleos explican su dependencia con las formas de trabajo urbano y la poca independencia que tienen para decidir sobre sus condiciones laborales. Sin embargo, las necesidades de reproducción de los grupos son de carácter inmediato, por ello, al no contar con una base material que les permita sostenerse y elegir libremente las opciones de empleo, se ven obligadas a mantenerse en condiciones de trabajo precarias.

Aunado a lo anterior, las ofertas laborales a las que pueden acceder estos grupos socialmente vulnerables, se localizan principalmente fuera de sus delegaciones de residencia y más ligados o cercanos al centro de la ciudad. Se observó que estos grupos son los que mayor desplazamiento laboral tienen, sobre todo los de la delegación Tláhuac, por lo tanto, los costos del traslado a los centros de trabajo (gasto en transporte, tiempos de recorrido, agotamiento físico) repercuten en dos sentidos: primero, la 
posibilidad de realizar una segunda actividad que complemente sus ingresos y, segundo, la mayor dependencia a las formas laborales de la ciudad cada vez más centradas en actividades terciarias o de servicios.

\section{Conclusiones}

La categoría de borde -como instrumento metodológico basado en el reconocimiento de la heterogeneidad, simultaneidad y coexistencia de procesos socioterritoriales-, permitió transitar de la escala regional a la microsocial, para identificar y reconocer la existencia de algunos grupos domésticos que están inmersos en una dinámica de vulnerabilidad social constante, al depender y condicionar sus formas de reproducción de una dinámica urbana externa y contigua al territorio en el que habitan.

La conjunción de factores como: la estructura del grupo doméstico, el origen del jefe o jefa de familia, la forma de acceso y el tipo de territorio en el que habitan, así como la actividad productiva, facilitan que los grupos domésticos de algunas localidades del borde sur de la ZMCM reproduzcan o no formas de vida socialmente vulnerables. Estos grupos, aportan mano de obra de baja calificación y depreciada remuneración a la ciudad y coexisten con multiplicidad de grupos domésticos de características diversas, dentro de espacios caracterizados por formas de tipo rural y de conformación dispersa, pero cercanos a centros urbanos más consolidados.

Se observó además que el hecho de no recibir equipamiento e infraestructura por parte de las unidades administrativas a las que pertenecen, los grupos domésticos de estas localidades son agentes que frenan en cierta medida la expansión de la urbe hacia la porción sur de la cuenca, por lo tanto, estos grupos, además de vivir bajo condiciones de vulnerabilidad social, están asumiendo el costo a través de sus condiciones de vida precarias, la expansión de la ciudad.

Este trabajo pretende, en primer lugar, la identificación y reconocimiento de la multiplicidad de agentes que coexisten dentro de estos espacios del borde sur, entre ellos los grupos socialmente vulnerables. Asimismo, se busca abonar a la discusión para que desde las políticas y planes de uso de suelo y desde los programas sociales del Gobierno del Distrito Federal se trate esta problemática al crear lugares (subcentros de trabajo cercanos al borde sur) de oportunidad laboral bien remunerada para jefas de familia, o impulsen el desarrollo de proyectos productivos gestionados por las mismas localidades con base en las necesidades y capacidades de los beneficiarios, se busca además, pensar en la posibilidad de ofrecer alternativas sustentables para mejorar la falta de infraestructura y servicios en las viviendas bajo una política estricta de ocupación del 
suelo que no comprometa los servicios ambientales del suelo de conservación.

Es importante también continuar este tipo de estudio en otros espacios limítrofes de la Ciudad de México como el oriente y poniente, en donde es muy probable que se encuentren también grupos domésticos en condiciones de vulnerabilidad social quizá matizada por otras variables socioterritoriales y diversidad ampliada de procesos que se desarrollan en los llamados bordes de la Zona Metropolitana de la Ciudad de México.

\section{Fuentes consultadas}

Adler de Lomnitz, Larissa (1997), Cómo sobreviven los marginados, Siglo XXI, México.

Barsky, Andrés (2013), “Gestionando la diversidad del territorio periurbano desde la complejidad de las instituciones estatales. Implementación de políticas públicas para el sostenimiento de la agricultura en los bordes de la región metropolitana de Buenos Aires (2000-2013)", tesis de doctorado, Facultad de Filosofía y Letras, Departamento de Geografía, Universidad Autónoma de Barcelona, Barcelona.

Cepal (Comisión Económica para América Latina y el Caribe) (1998), "La exclusión social de los grupos pobres en Chile (LC/R.1824)", Santiago de Chile, <http://repositorio.cepal.org/handle/11362/ 31091>, 22 de febrero de 2014.

Cruz-Rodríguez, María Soledad (coord.) (2012), Periferias metropolitanas. Politicas públicas y medio ambiente, Universidad Autónoma Metropolitana unidad Atzcapotzalco-Red Nacional de Investigación Urbana, México.

De Grammont, Hubert C. (2005), “Prólogo”, en Héctor Ávila-Sánchez (coord.), Lo urbano-rural, ¿nuevas dimensiones territoriales?, Centro Regional de Investigaciones Multidisciplinarias-Universidad Nacional Autónoma de México, Cuernavaca, pp. 11-18.

De Oliveira, Orlandina, Mirielle Pepin y Vania Salles (comps.) (1989), Grupos domésticos y reproducción cotidiana, El Colegio de MéxicoUniversidad Nacional Autónoma de México-Porrúa, México. 
Fernández-Silva, Perla Yannelli (2011), "La relación campo-ciudad en las localidades del borde sur de la Zona Metropolitana del Valle de México", tesis de doctorado, Universidad Autónoma Metropolitana unidad Xochimilco, México.

García, Brígida, Héctor Muñoz y Orlandina de Oliveira (1989), "Familia y trabajo en México", en De Oliveira, Orlandina, Mirielle Pepiny Vania Salles (comps.), Grupos domésticos y reproducción cotidiana, El Colegio de México-Universidad Nacional Autónoma de México-Porrúa, México, pp. 487-507.

Gobierno del Distrito Federal-Secretaría del Medio Ambiente-Comisión de Recursos Naturales (2000), "Programa General de Ordenamiento Ecológico del Distrito Federal, Manual”, Gobierno del Distrito Federal-Secretaría del Medio Ambiente-Comisión de Recursos Naturales, México.

Inegi (Instituto Nacional de Estadística y Geografía) (2010), Censo de Población y Vivienda 2010. Resultados definitivos, Inegi, México.

Inegi (Instituto Nacional de Estadística y Geografía) (2005), "II Conteo de población y Vivienda 2005. Principales resultados por localidad", Inegi, México.

Inegi (Instituto Nacional de Estadística y Geografía) (2000), Censo de población y Vivienda 2000. Resultados definitivos, Inegi, México.

Massey, Doreen (2005), For space, London, Sage Publications.

Méndez-Sastoque Marlon (2005), “Contradicción, complementariedad e hibridación en las relaciones entre lo rural y lo urbano", en Héctor Ávila-Sánchez (coord.), Lo urbano-rural, ¿nuevas expresiones territoriales?, Universidad Nacional Autónoma de México- Centro Regional de Investigaciones Multidisciplinarias, Cuernavaca, pp. 87-122.

Negrete-Salas, Ma. Eugenia (2000), “Dinámica demográfica”, en Gustavo Garza (coord.), La ciudad de México en el fin del segundo milenio, Gobierno del Distrito Federal-Colegio de México, México, pp. 247-255. 
Pacheco-Ladrón de Guevara, Lourdes (2002), "La rurbalidad", Ciudades, núm. 54, Red Nacional de Investigación Urbana, Puebla, pp. 55-58.

Pizarro-Hofer, Roberto Guillermo (2001), La vulnerabilidad social y sus desafios: una mirada desde América Latina, Comisión Económica para América Latina y el Caribe, Organización de las Naciones Unidas, Santiago de Chile.

Pradilla-Cobos, Emilio (2011), Ciudades compactas, dispersas, fragmentadas, Universidad Autónoma de México unidad Xochimilco-Miguel Ángel Porrúa, México.

Pradilla-Cobos, Emilio (2002), "Campo y ciudad en el capitalismo actual”, Ciudades, núm. 54, Red Nacional de Investigación Urbana, Puebla, pp. 3-8.

Ramírez-Velázquez, Blanca Rebeca (2012), "La vulnerabilidad territorial del neoliberalismo mexicano", en José Luis Calva-Tellez (coord.), Desarrollo regional y urbano. Análisis estratégico para el desarrollo, vol. 13, Ed. Juan Pablos-Consejo Nacional de Universidades, México, pp. 251-272.

Ramírez-Velázquez, Blanca Rebeca (2007), "Del suburbio y la periferia al borde: el modelo de crecimiento de la Zona Metropolitana del Valle de México (ZMVM)", Banlieues et périphéries des métropoles latino-américaines. L'Ordinaire latino-américain, núm. 207, Institut pluridisciplinaire pour les études sur les Amériques à Toulouse, Toulouse, pp. 69-89.

Ramírez-Velázquez, Blanca Rebeca y Patricia Arias (2002), "Hacia una nueva rusticidad", Ciudades, 54 (14), Red Nacional de Investigación Urbana, Puebla, pp. 9-14.

Skewes, Juan Carlos (2014), "Poblaciones agredidas, disputas urbanas y escenarios posibles: la producción de la ciudad desde las periferias", Revista Invi, 81 (29), Universidad de Chile, Santiago de Chile, pp. 9-17.

Villarreal-González, Diana Rebeca y Víctor Castañeda (1986), Urbanización y autoconstrucción de vivienda en Monterrey, Centro de Ecodesarrollo, México. 
Recibido: 9 de septiembre de 2014. Corregido: 26 de junio de 2015. Aceptado: 3 de febrero de 2016.

Perla Yannelli Fernández-Silva. Mexicana. Arquitecta por la Universidad Iberoamericana; maestra en ciencias en recursos naturales y desarrollo rural por El Colegio de la Frontera Sur; investigadora asociada en Proyectos de colonias indígenas en la periferia de la ciudad de San Cristóbal de las Casas, Chiapas. Es doctora en ciencias sociales con especialidad en estudios socio-territoriales por la Universidad Autónoma Metropolitana, México. Actualmente se encuentra en estancia Posdoctoral de Conacyt en el Programa de Posgrado en Urbanismo de la UNAM. Su línea de investigación actual es desarrollo regional del noroeste de México, y desarrolla el proyecto de investigación: "Formas de apropiación productiva y usos del territorio en ejidos de Puerto Peńasco, Sonora”. Actualmente realiza una estancia posdoctoral en el Programa de Posgrado de Urbanismo de la UNAM y desarrolla el proyecto de investigación: "Eco-tecnologías para la vivienda en la Ciudad de México". Sus últimas publicaciones: "El suelo de conservación de Ciudad de México. Implicaciones en la vida de sus habitantes", Anuario de Investigación del Posgrado de Urbanismo, 3 (3), Universidad Nacional Autónoma de México, México, pp. 147-170 (2016) (en prensa). En coautoría: "De la periferia al borde: condiciones de trabajo de las familias rurales del borde sur de la Zona Metropolitana de la Ciudad de México", en H. Salas y Alejandra Toscana (coords.) Territorios $y$ Regiones, vol. VII, Asociación Mexicana de Estudios Rurales, Universidad Nacional Autónoma de México, México, pp. 118-137 (2015); "Pobreza urbana, otra lectura de la pobreza”, en Adrián Guillermo Aguilar (coord.), La Ciudad de México en el siglo XXI, Porrúa, México, pp. 277-284 (2016) (en prensa).

Blanca Rebeca Ramírez-Velázquez. Mexicana. Es geógrafa y doctora en urbanismo por la Universidad Nacional Autónoma de México, con estudios de maestría en planeación regional en la Universidad de Aberdeen, Gran Bretaña. Concluyó el diplomado de estudios a profundizar en la Universidad de París III, Sorbona. Profesora titular C, tiempo completo del Departamento de Teoría y Análisis de la Universidad Autónoma de México unidad Xochimilco desde 1989. Es miembro del Sistema Nacional de Investigadores, nivel II, autora de 10 libros y 95 artículos y capítulos de libros sobre temas urbano-regionales. Es co-coordinadora de los libros Debates y estudios de la movilidad laboral en la región centro del pais: alcances y dimensiones desde México, Universidad Autónoma de México, México (2016); Espacio, paisaje, región, territorio y lugar: La diversidad en 
el pensamiento contemporáneo, UNAM-Universidad Autónoma de México, México (2015) y Teorias sobre la ciudad en América Latina, Universidad Autónoma Metropolitana (2013). 